\title{
Phenolic Foam Based the Influence of Intumescent Flame Retardancy System Ammonium Polyphosphate/Pentaerythritol/Melamine
}

\author{
Hui-lu $\mathrm{Li}^{1}$, Cui Huo ${ }^{2,{ }^{*}}$ Peng Miao ${ }^{3}$, Ting Zhang ${ }^{4}$, and Hong-li Wei ${ }^{5}$ \\ ${ }^{1}$ College of Materials Science and Engineering,Xi'an University of Science and Technology, Xi'an \\ 710054, China \\ ${ }^{2}$ College of Materials Science and Engineering,Xi'an University of Science and Technology, Xi'an \\ 710054, China \\ ${ }^{3}$ College of Materials Science and Engineering, Chang'an University, Xi'an 710054, China \\ College of Materials Science and Engineering,Xi'an University of Science and Technology, \\ Xi'an 710054, China
}

Keywords: Flame Retardant, Ammonium Polyphosphate, Melamine, Pentaerythritol, Oxygen Index

\begin{abstract}
Phenolic foam with high flame retardation was prepared through intumescent flame retardant treatment with physical blending of ammonium polyphosphate(APP) 、 pentaerythritol(PER)、melamine(MIR). Test results showed that the phenolic foam achieves high flame retardation when $\mathrm{APP} / \mathrm{PER} / \mathrm{MIR}=4: 1: 0.5$ (dosage: $25 \%$ ). The thermal conductivity, compressive strength and oxygen index (LOI) of the material were $0.03 \mathrm{w} / \mathrm{m} . \mathrm{k}, 0.15 \mathrm{Mpa}$ and $83 \%$, respectively. The thermal weight loss at $750{ }^{\circ} \mathrm{C}$ was $76 \%$ and the maximum service temperature was $180^{\circ} \mathrm{C}$. Moreover, flame-retardant mechanism of the fire retardant was analyzed.
\end{abstract}

\section{Introduction}

"11.15" Shanghai jiaozhou road big fire [1] and On February 9, 2009, CCTV fire events[2] .All this fire incidents of external wall insulation finally put forward higher requirements[3-5].Polystyrene (PS) thermal insulation material is widely used as external wall insulation materials[6]. Burning PS will generate black smoke and molten drops and is toxic[7-8], which will cause great harms to life. Therefore, it is urgent to develop high-effective thermal insulation materials with high flame retardation[9-10]. APP, PER and MIR, serving as the protonic acid source, carbon skeleton and air source of the flame retardant, all belong to the chemical intumescent flame retardant system. Under certain temperature, molecular interactions make PER molecules carbonized under the effect of the strong acid APP, forming a charring layer that could stop fire. Finally, MIR produces ammonia gas through thermolysis. This reduces contact area between oxygen and the phenolic foam and envelops the charring layer within the phenolic foam surface, thus realizing the goal of flame retardation[11-12]. The intumescent flame retardant system is widely used in PP, PVC, PET, leather and flame retardant coating, and achieves great effect[13-14]. However, few reports on its application in phenolic foam have been reported yet[15].

In this paper, effect of composition proportion of the intumescent flame retardant system on flame retardation of phenolic foam was discussed. Test results showed that flame retardant treatment could increase LOI, TG and temperature resistance of the phenolic foam significantly. This is of important significance for the development and application of exterior insulation materials. 


\section{Experiment}

\section{Reagent and Instrument}

\section{Test Reagent}

Phenolic resin (industrial grade): Jinan Zhangqiu Chemical Industrial Park; water soluble silicone oil (industrial grade); P-toluene sulfonic acid (chemical grade); phosphoric acid (industrial grade); N-pentane (analytical grade); APP (industrial grade); MIR (industrial grade); PER (analytical grade).

\section{Test Instrument}

Test instruments include DRY-300F thermal conductivity tester (Tianjin Fred Technical Co., Ltd), CTM2500 microcomputer control electronic universal material testing machine (Xieqiang Instrument Manufacturing (Shanghai) Co., Ltd), JF-3 LOI tester (Nanjing Jiangning Analysis Instrument Plant), TGA Q50 thermal analyzer (TA Company, USA), and infrared spectrometer (Brooker, Germany).

\section{Preparation Technique of Flame-retardant Phenolic Foam}

\section{Preparation of Flame-retardant Phenolic Foam}

Phenolic resin and surface active agent were mixed completely under room temperature and then emulsified for $5 \mathrm{~min}$. Next, fire retardants were added successively and mix them evenly. Finally, curing agent, foaming agent and emulsified flame retardants were dispersed quickly for $0.5-1 \mathrm{~min}$, and then pour them into a self-made $200 * 200 * 20 \mathrm{~mm}$ mould immediately. A piece of flame-retardant phenolic foam board with a certain geometric size will be formed under $65-85^{\circ} \mathrm{C}$.

\section{Preparation of Curing Agent}

Weigh 6-8 units of phosphoric acid, 8-10 units of P--toluene sulfonic acid and 8-10 units of distilled water. Mix them evenly and then pour into the mould in the same way stated in Section 2.2.1. Finally, they will foam into curing agent in the mould. The formula of preparing flame-retardant phenolic foam. As is shown in table 1.

Table 1 the formula of preparing flame-retardant phenolic foam

\begin{tabular}{lc}
\hline Sovent & Content \\
\hline Phenolic Resin & 100parts \\
Surfactants & 2-13parts \\
Vesicant & 15-25parts \\
Curing agent & 18-30parts \\
Fire Retardants & 10-30parts \\
\hline
\end{tabular}

\section{Properties Characterization}

\section{LOI}

LOI was tested by TB/T 2701-1996. It is the minimum oxygen concentration (volume percentage) needed for the test sample to maintain balance burning in nitrogen and oxygen mixture. Sample size is $5 \mathrm{~mm} \times 5 \mathrm{~mm} \times 100 \mathrm{~mm}$.

\section{Heat Conductivity}

Heat conductivity was tested by the DRY-300F thermal conductivity tester (Tianjin Fred Technical Co., Ltd). Heat conductivity of samples was calculated by testing temperature difference between the hot plate and cold plate with a double-specimen test device. Sample size is $200 \mathrm{mx} 200 \mathrm{~m} \times 20 \mathrm{~m}$.

\section{FTIR Analysis}


FTIR analysis used TENSOR27 (Brooker, Germany).

\section{TGA}

TGA was implemented on the 150 thermal analyzer (TA Company, USA). Temperature rises from room temperature to $800{ }^{\circ} \mathrm{C}$ at a rate of $10{ }^{\circ} \mathrm{C} / \mathrm{min}$, with $\mathrm{N} 2$ protection.

\section{Results and Discussion}

It can infer from chemical structural theory of APP and PER that when end groups of one APP molecule needs 0.25 PER molecule, the molecular structure is three-dimensional, which has the best flame retardation effect. To verify this theory, LOI and heat conductivity of the phenolic foam under different dosages of APP and PER (5\%, 10\%, 15\%, 20\% and 25\%) and different APP/PER (4, 2, 1, $0.5)$ were analyzed.

\section{Effect of Different APP/PER and Dosages on LOI of Phenolic Foam}
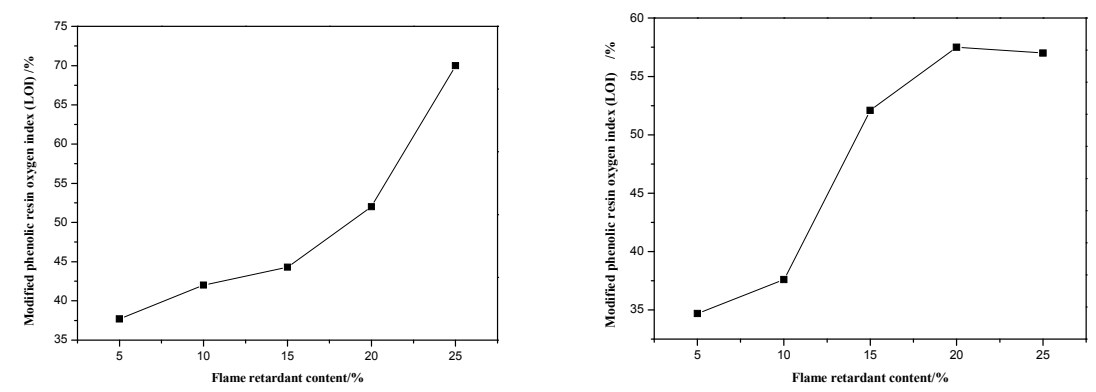

(a)

(b)
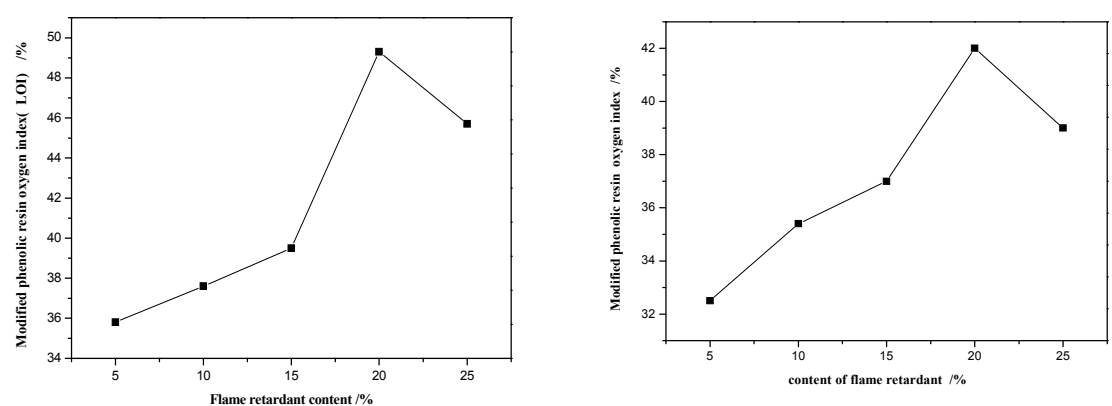

(c)

(d)
(a) $-\mathrm{m}_{(\mathrm{APP})}: \mathrm{m}_{(\mathrm{PER})}=4$
(b) $-\mathrm{m}_{(\mathrm{APP})}: \mathrm{m}_{(\mathrm{PER})}=2$
(c) $-\mathrm{m}_{(\mathrm{APP})}: \mathrm{m}_{(\mathrm{PER})}=1$
$(\mathrm{d})-\mathrm{m}_{(\mathrm{APP})}: \mathrm{m}_{(\mathrm{PER})}=0.5$

Fig.1 Effect of different APP/PER and dosages on LOI

In Fig.1, LOI of the phenolic foam increases continuously as dosage of flame retardant system increases. The maximum LOI is achieved at 25\% of flame retardant system (Fig.1-(1)), reaching 70\%. The maximum LOI in Fig.1-(2), (3) and (4) is $45.7 \%, 57 \%$ and $41.1 \%$, respectively. Viewed from LOI, the phenolic foam achieves the best flame retardation effect when APP/PER $=4$. Analyzed from theory and practical situation, pyrophosphoric acid produced by APP decomposition could combine well with alcoholic hydroxyl group in PER, forming a carbon layer that has strong fire retardation. In Fig.1-(2), (3) and (4), although LOI increases in general, it can infer from test data that the formed carbon layer is not so dense as that in Fig.1-(1), thus resulting in the relatively lower LOI. LOI of unprocessed phenolic foam generally ranges between $28-36 \%$. The flame retardation of the modified phenolic foam improves significantly. The theoretical calculation agrees with test results, confirming 
the feasibility of the theory. This is very important to fire prevention of phenolic foam plastics. Therefore, LOI of the phenolic foam reaches a high value when APP/PER=4.

\section{Effect of MIR Content on LOI of Phenolic Foam}

In Fig. 1, it is determined that flame retardation of phenolic foam when APP/PER $=4$ reaches $70 \%$. MIR serves as the air source in the intumescent flame retardant system. In physical blending, inadequate MIR content makes it impossible to form a continuous carbon layer on the phenolic foam surface, thus decreasing its flame retardation. However, excessive MIR content will make the formed carbon layer blown overflow by the ammonia gas decomposed from MIR, which also will decrease flame retardation of the phenolic foam. Therefore, it is very important to choose appropriate MIR content.

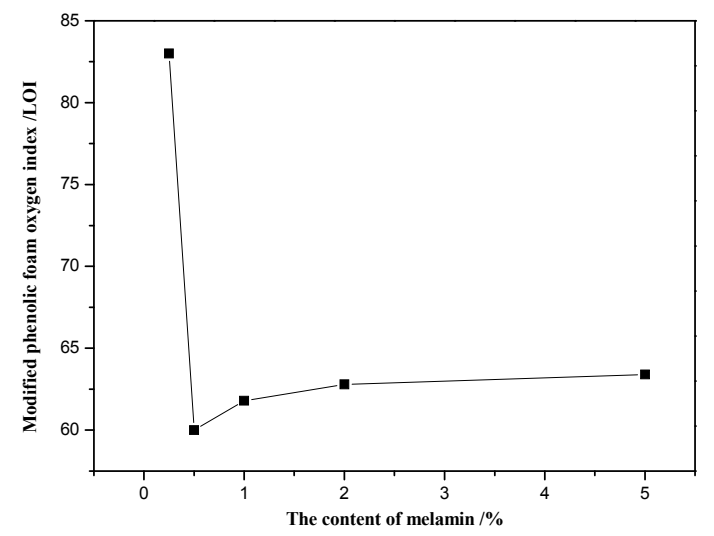

Fig.2 Effect of MIR percentage on LOI of flame-retardant phenolic foam

It can be seen from Fig.2 that LOI of the flame-retardant phenolic foam drop dramatically and then becomes relatively stable with the increase of MIR content. When MIR content is $0.25 \%$, LOI reaches as high as $83 \%$. With the continuous increase of MIR content, thermolysis of MIR is very quick and non-flammable gases escape quickly, which goes against flame retardation of the phenolic foam. According to test data, the appropriate MIR content is $0.25 \%$. At this moment, non-flammable gases are absorbed surrounding air holes of the phenolic foam, which separate oxygen from the foam, stopping fire burning.

\section{Effect of Different APP/PER and Flame Retardant Contents on Heat Conductivity of Phenolic Foam}

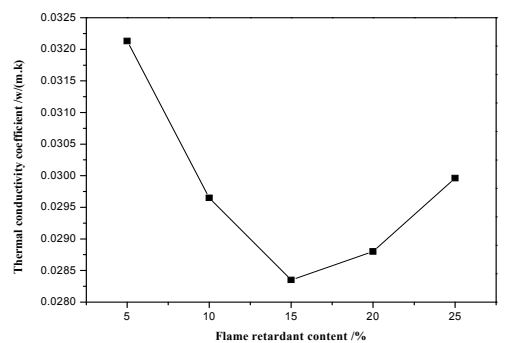

(a)

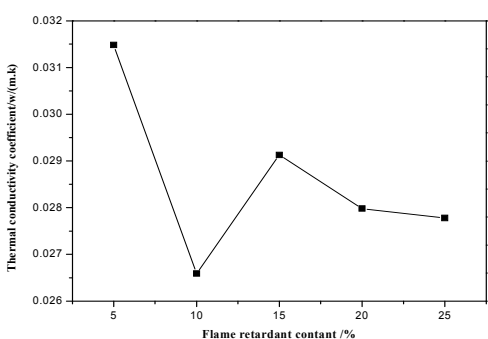

(b) 


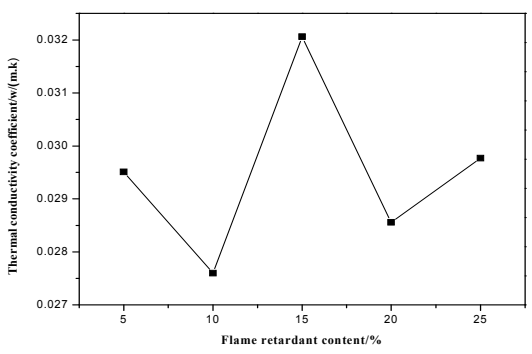

(c)

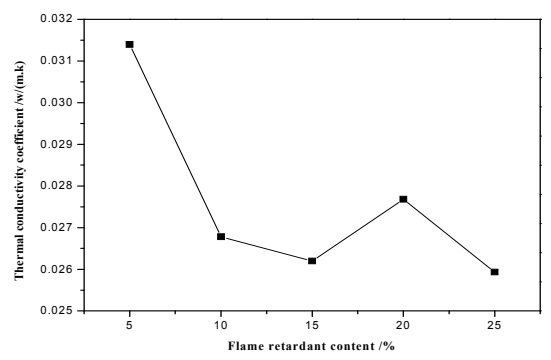

(d)

$\mathrm{a}-\mathrm{m}_{(\mathrm{APP})}: \mathrm{m}_{(\mathrm{PER})}=4 \quad \mathrm{~b}-\mathrm{m}_{(\mathrm{APP})}: \mathrm{m}_{(\mathrm{PER})}=2 \quad \mathrm{c}-\mathrm{m}_{(\mathrm{APP})}: \mathrm{m}_{(\mathrm{PER})}=1 \quad \mathrm{~d}-\mathrm{m}_{(\mathrm{APP})}: \mathrm{m}_{(\mathrm{PER})}=0.5$

Fig.3 Effect of different APP/PER and flame retardant contents on heat conductivity

Fig. 3 shows that when adding 5\% intumescent flame retardant, heat conductivity of the phenolic foam is basically $0.03 \mathrm{w} /(\mathrm{m} . \mathrm{k})$. With the increase of flame retardant content, heat conductivity of the phenolic foam reduces slightly, about $0.027-0.028 \mathrm{w} /(\mathrm{m} . \mathrm{k})$. This may be because that when increasing flame retardant, it opens more paths for heat transmission or the flame retardant may absorb heats to a certain extent. Based on comprehensive comparison, increase of flame retardant within a certain range won't influence heat conductivity significantly.

\section{Effect of Flame Retardant Content on Dompressive Strength of the Phenolic Foam When} $\mathbf{A P P} / \mathbf{P E R}=4$

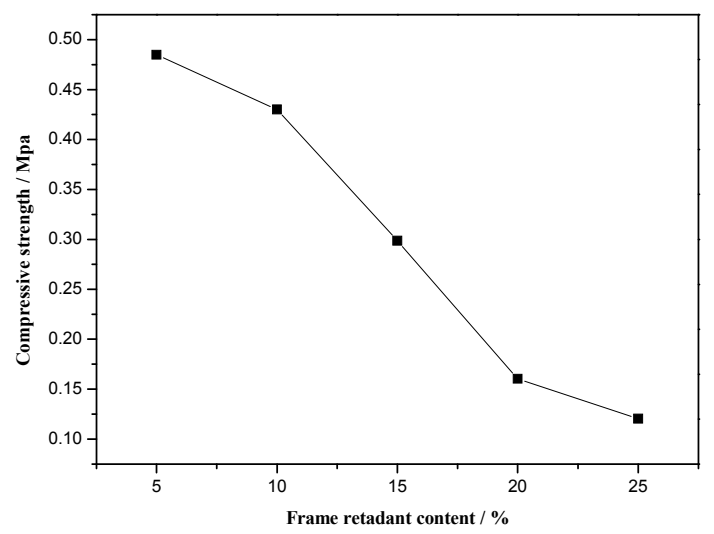

Fig.4 Effect of flame retardant content on compressive strength of the phenolic foam

It can be seen from Fig.4 that with the increase of flame retardant, compressive strength of the flame-retardant phenolic foam decreases continuously. The compressive strength is $0.48 \mathrm{Mpa}$ at $\%$, but reduces sharply to $0.12 \mathrm{Mpa}$ at $25 \%$, basically meeting the requirement of GB/T8813-1988. Due to the micro dosage of flame retardant, $5 \%$ is conducive to strengthen structure of the phenolic foam. Further increase of flame retardant will increase specific surface area and volume occupancy of nanoparticles, thus reducing strength of the phenolic foam.

\section{FTIR of the Flame-retardant Phenolic Foam}




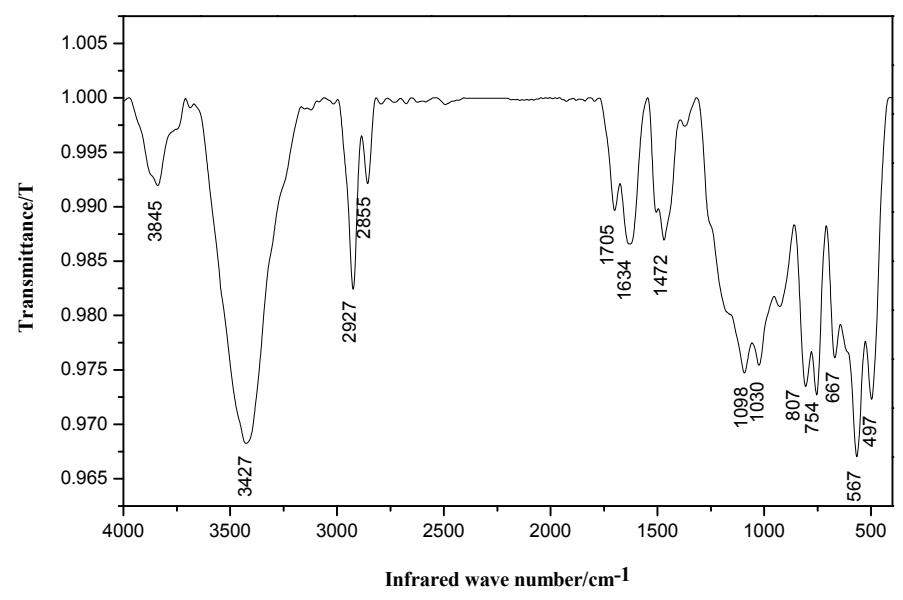

Fig.5 FTIR of flame-retardant phenolic foam when APP/PER=4 and $25 \%$ dosage of flame retardant

In Fig.5, there's a strong stretching vibration peak of C-H on benzene ring at $2927 \mathrm{~cm}^{-1}$. Peakes at $807 \mathrm{~cm}^{-1}$ and $754 \mathrm{~cm}^{-1}$ are tri-substituted peaks in the phenolic resin, which indicates that the flame-retardant phenolic resin is solidified basically completely. There's a N-H absorption peak at $3427 \mathrm{~cm}^{-1}$. Since APP doesn't involved in the reaction before burning of physical add reaction, it can deduce that the phenolic foam contains APP and MIR. Peaks at $1030 \mathrm{~cm}^{-1}$ and $1098 \mathrm{~cm}^{-1}$ belong to absorption vibration peaks of C-O-C and P-O, indicating the existence of PER. Peaks at $1634 \mathrm{~cm}^{-1}$ and $1705 \mathrm{~cm}^{-1}$ are vibration peaks of $\mathrm{C}=\mathrm{C}$ of benzene ring. It basically could determine that all flame retardants have been added through characteristic absorption peaks of groups in the FTIR.

\section{TGA of the Flame-retardant Phenolic Foam}

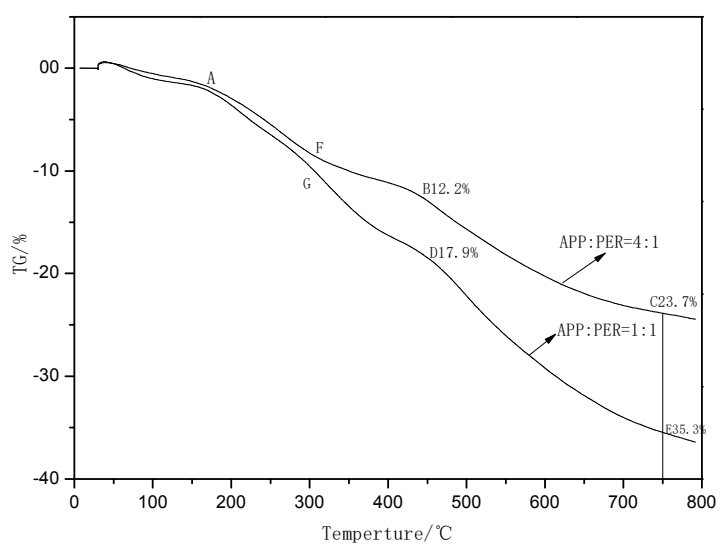

Fig.6 TG curve of the flame-retardant phenolic foam when $25 \%$ dosage of flame retardant

It can be seen from Fig.4 that the system is more stable to heat when APP: PER = 4: 1 . As temperature rises continuously, mass of the flame-retardant phenolic foam decreases continuously. Different APP/PER have a great value on TG of the flame-retardant phenolic foam. The mass remains basically before the A point, which is mainly caused by volatilization of water and small molecule overflow in the phenolic foam. When temperature reach to $F$ and $G$ points, mass loss of the flame-retardant phenolic foam reaches about $10 \%$. This section can be divided into two parts. The first part is crosslinking association polycondensation of hydroxymethyl on the benzene ring to precipitate water, which is known as thermocuring reaction. The second part is thermolysis of APP to release water molecules and ammonia. The APP will produce polyphosphoric acid which will make esterification reaction with PER. When temperature reach to B and D points, the mass loss are $12.2 \%$ 
and $17.9 \%$ respectively. This is caused by mutual condensation of methylene groups of the phenolic resin and thermolysis of the flame retardant system which is accompanied with some decomposition. In the $\mathrm{BC}$ and $\mathrm{DE}$ sections, molecular chain in the flame-retardant resin splits under temperature migration. Some volatile gases are produced, such as $\mathrm{H} 2 \mathrm{O}, \mathrm{CO} 2, \mathrm{CH} 4, \mathrm{NH} 3$, etc., including decomposition of MIR. This period is the quick carbonization of the flame-retardant phenolic resin, forming a dense carbon layer. After $\mathrm{C}$ and $\mathrm{E}$ points, the curve is basically stable, indicating that small molecules generated through molecular decomposition are volatilized completely. Rest mass basically belongs to residual carbon. GB/T5464-2010 stipulates explicitly that more than 50\% residual carbon in exterior insulation material in air under $750{ }^{\circ} \mathrm{C}$ could meet requirement basically.

\section{Establishment of Flame Retardant Model}

The left picture in Fig. 7 is the flame-retardant phenolic foam plastic. Exposed to irradiation, heat or open fire, the flame retardant APP will decompose non-flammable gases immediately and form a non-flammable protection layer surrounding the foam. while generating a strong acid, which captured hydrogen in the foam. Under the further effect of heat, it will make aromatization and forms carbon layer with a black center. This carbon layer could stop fire and form carbons. In this way, flame retardents APP, PER and MIR all could develop their fire prevention and carbon formation effect, thus increasing residual carbon and combustion time of the phenolic foam. This provides confirmatory analysis to further study A2 fireproof thermal insulation materials.

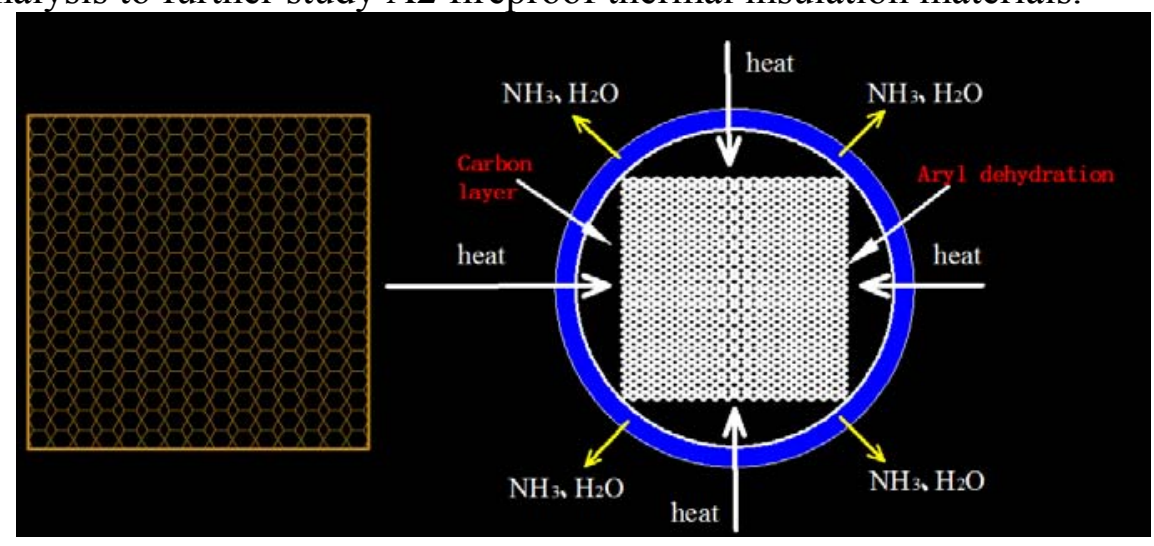

Fig.7 APP/PER/MIR flame retardant model mechanism

\section{Conclusions}

(1) Flame retardant treatment of the phenolic foam with physicochemistry intumescent flame retardant system could increase its fire resistance to a large extent.

(2) Adding a certain flame retardant system through physical blending could improve the comprehensive property of the phenolic foam, such as heat conductivity and mechanical properties.

(3) The appropriate flame retardant treatment to phenolic foam is $25 \%$ content of flame retardant system and APP/PER/MIR=4:1:0.5. The phenolic foam processed by such flame retardant system could meet GB/T8464-2006 in term of comprehensive performance.

(4) According to test data analysis, it is of important significance to make exterior insulation materials to reach A2 fireproof level of GB/T8464-2006 through flame retardant treatment of the phenolic foam with intumescent system while maintaining the basic fireproof level.

\section{Acknowledgement}

This research was financially supported by the Natural Science Foundation of China. 


\section{References}

[1] Yin Y C, The application of the phenolic foam at home and abroad, the wall heat preservation[J] China Building Materials News 2011, 6: 1-2.

[2]Yang J P. Phenolic foam board exterior wall insulation system product introduction - a kind of excellent fireproof performance of heat preservation system Cutting-edge engineering materials co, LTD, Shanghai,

[3] Xu XQ.Building exterior insulation materials combustion performance and countermeasures[J] applied technology,2011,27(6):99-100.

[4] Nazım Usta. Investigation of Fire Behavior of Rigid Polyurethane Foams Containing Fly Ash and Intumescent Flame Retardant by Using a Cone Calorimeter [J]. Journal of Applied Polymer Science, Vol. 124, 3372-3382(2012).

[5] David Eaves.Handbook of polymer foams[M].Rapra Technology,2004,1-7.

[6]The ministry of public security, housing and urban-rural development. Civil building outside wall thermal insulation system and outside wall decoration of fire prevention regulations. The ministry of public security of housing and urban-rural development and word [2009] no. 46, 2009,9,25.

[7]Jongmin Kim, Jae-Hyug Lee,Tae-Ho Song.Vacuum insulation properties of phenolic foam[J]. International Journal of Heat and Mass Transfer 55 (2012) 5343-5349.

[8] Tao S J, "File 46" The general idea is correct [J] .Special Cover, 2010,13-14.

[9]Bu Gi Kim, Dai Gil Lee. Development of microwave foaming method for phenolic insulation foams[J]. Journal of materials processing technology 201 (2008)716-719.

[10]Liu Y, Jia R L etc. Polyethylene with flame retardants and their research progress of combined systems [J]. Polymer bulletin, 2006,78-79.

[11] Bao Z Y, Dong Y M, IFR technology and application [M] Harbin: Harbin Institute of Technology Press, 2005.

[12] Ou Y X, Practical flame retardant technology. Beijing: chemical industry press, 2002.

[13] Li G X, Liang G Z. Study on APP/PER/MEL flame retardant coating modified by MoO3 and expandable grapiite[J].Modern Chemical industry.Vol,26,31-33.

[14]Wang D C, Chang G W, Chen Y. Preparation and thermal stability of boron-containing phenolic resin/clay nanocomposites[J]. Polymer Degradation and Stability 93 (2008),125-133.

[15] Yamashita Y,Ouchik.A study on carbonization of phenol-formaldehyde resin labeled with deuterium and13C[J].Carbon,1981,19(2):89-94. 